\title{
Optimal and Non-Optimal Based Feedback Switching Control Techniques for the Turbocharged Diesel Engines
}

\author{
Shashidhar S Gokhale ${ }^{1}$, Sudarshan Patil Kulkarni ${ }^{2}$, Yathisha L ${ }^{3}$ \\ Research Scholar, JSSRF, Mysuru, Karnataka, India - $570006^{1}$ \\ Associate Profesor, Dept. of ECE, ATME College Of Engineering, Mysuru, Karnataka, - 570 028, India ${ }^{1,3}$ \\ Professsor, Dept. of ECE, JSSRF \& JSSTU, Mysuru, Karnataka, India - $570006^{2}$, \\ Email: shashisg@gmail.com ${ }^{1}$,sudarshan_pk@sjce.co.in ${ }^{2}$, yathisha.171@gmail.com ${ }^{3}$
}

\begin{abstract}
Switching between two feedback control strutcures ia an essential parameter in today's scenario of automotive systems to enhance the system performance, safe operation during the component failures in any of the control structures, environmental aspects, etc. Hence, in this paper two standard switching control algorithms are applied and compared for the dynamics of turbocharged diesel engines. Later, in the practical scenario, due to environmental and other factors, there is a possibility of system becoming unstable. To overcome this drawback, this paper also introduces intelligent existing switching control algorithm to switch between one optimal control (stable) and other with non-optimal control (unstable) to obtain overall performance of the closed loop system to be stable.
\end{abstract}

Keywords - LQR; Switching Control; EGR; Optimal Control

\section{INTRODUCTION}

The vital part of any automotive vehicle is the engine. Today's challenge for the vehicle industry is to develop energy efficient engines. An engine which generates power on its own is referred to as automotive engine. Automotive engines are generally classiffied as internal combustion and external combustion engines. In internal combustion engines, Exhaust Gas Recirculation (EGR) is a Nitrogen Oxide (NOx) emissions reduction technique used in gasoline/petrol and diesel engines. EGR works by recirculating a portion of an engine's exhaust gas back into the engine cylinders (Engine Manifold) where combustion takes place.

(Magdi S. Mahmoud et al. 2012) presented linear turbocharged diesel engine model and controllers are designed using the linear-quadratic regulator (LQR), linear-quadratic Gaussian regulator (LQGR), $\mathrm{H}_{2}, \mathrm{H}_{\infty}$ and mixed $\mathrm{H}_{2} / \mathrm{H}_{\infty}$. The MATLAB simulation results of all the proposed controllers are compared and it yields that the mixed $\mathrm{H}_{2} / \mathrm{H}_{\infty}$ provides best performance compared to all other controllers. A novel control design procedure for air management and fueling strategy (AMFS) of diesel engines in lights of a multi-layer control structure (MLCS) was proposed by (Adrian Ilka, Nikolce Murgovski \& Jonas Fredriksson et al. 2019). The suboptimal gain scheduled Proportional Integral Derivative (PID) controllers are developed for the model to anlyze the stability.

(Felipe Castillo Buenaventura \& Emmanuel Witrant et al.2015) describes a novel technique to control the air fraction in the intake of dual-loop exhaust gas recirculation (EGR) diesel engines. The LQR control approach has been introduced for the air

fraction engine model and the simulation results reveal that better performances are obtained with the proposed approach. The state feedback control approach has been developed for the control of diesel engine EGR system by (Tianpu Dong \& Fujun Zhang et al.2015). The experimental results were compared with conventional PID Controllers and it reveal that more rapid dynamic response and smaller overshoots in transient control are achieved with the proposed state feedback control.

Switched systems are composed of a group of sub-systems guided by a switching law that governs the change among these subsystems. In LTI systems, by intelligently switching between a number of control structures depending on the state value, improved performance can be achieved compared to individual control structure. Hybrid or switching systems, commonly found in control theory are characterised by combination of both continuous and discrete systems and they have created an enormous growth of interest in dynamical systems. The performance of these systems can be realised by switching between relatively two simple linear time invariant (LTI) systems with different control techniques (Keith R Santarelli et al.2008, 2009 \& 2010).

The advantage of switching between two feedback controllers is to combine the properties of both and to introduce a new property that is not present in any of the feedback controllers. (Zhi Hong Huang \& Cheng Xiang et al. 2007) derived a necessary and sufficient condition for stability of arbitrarily switched second order LTI systems with marginally stable subsystems. The performance based switching algorithm for LTI systems based on Lyapunov stability criteria was developed by (Lalitha S Devarakonda et al. 2005 \& 2006). Switching control strategy is proposed for the UPFC, based on three scenarios involving high, low and normal conditions by (Yathisha L \& S Patil Kulkarni et al. 2015 \& 2017).

In the present work, the feedback controllers are optimized using LQR theory and the existing switching control techniques are suitably modified and developed for the turbocharged diesel engine model and the results are compared for the selection of better algorithm in future as a first part. Later, the feedback controllers are designed using optimal (Stable Controller) \& non-optimal (Unstable controller) and the intelligent existing switching control technique is suitably modified and applied to show that the overall system will achieve stability. 


\section{Available online at www.ijrat.org}

The remainder of the paper is organized as follows. Section II describes Linear Time Invariant (LTI) model of Turbocharged diesel engine dynamics with EGR system. Optimal control theory and the switched linear control theory are respectively discussed in Sections III and IV. Simulation results and discussions are presented in Section V. Section VI concludes the paper.

\section{DYNAMIC MODEL}

The diesel engine system consists of the intake manifold, exhaust manifold, turbocharger, EGR valve, intercooler, EGR cooler and cylinder, as shown in Fig. 1.

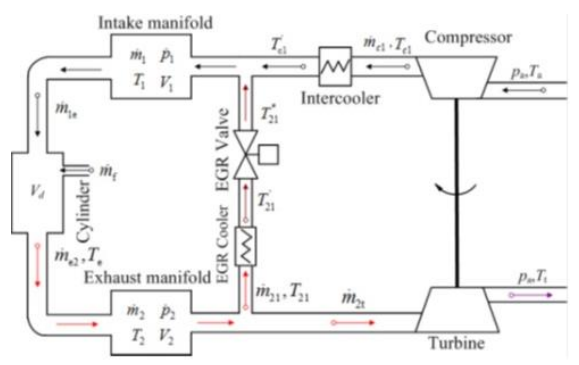

Fig. 1. Block diagram of turbocharged diesel engine with HP EGR system

The analysis of air system can be desribed by seven differential equations:

$$
\begin{gathered}
\dot{M}_{1}=\dot{M}_{c 1}+\dot{M}_{21}-\dot{M}_{1 e} \\
\dot{P}_{1}=\frac{\gamma R_{g}}{V_{1}}\left(\dot{M}_{c 1} T_{C 1}^{\prime}+\dot{M}_{21} T_{21}^{\prime}-\dot{M}_{1 e} T_{1}\right) \\
\dot{F}_{1}=\frac{\dot{M}_{21}\left(F_{2}-F_{1}\right)+\dot{M}_{c 1}\left(1-F_{1}\right)}{M_{1}} \\
\dot{M}_{2}=\frac{\dot{M}_{e 2}-\dot{M}_{21}-\dot{M}_{2 t}}{\dot{P}_{2}}\left(\dot{M}_{e 2} T_{e}+\dot{M}_{21} T_{2}-\dot{M}_{2 t} T_{2}\right) \\
\dot{P}_{c}=\frac{1}{\tau_{t c}}\left(n_{M} P_{t}-P_{c}\right)
\end{gathered}
$$

Tha above model can be simplified by selecting the state variables $x=\left[P_{1}, P_{2}, X_{0,1}, X_{0,2}, P_{c}\right]$ and the input variable $u=u_{\text {egr }}$, the state space equation of the air system can be derived as:

$$
\dot{P}_{1}=-K_{1} P_{1}+K_{2} C_{d}\left(U_{e g r}\right) \Psi_{e} P_{2}+\frac{K_{3}}{\left(\pi_{c}-1\right)} P_{c}
$$

$$
{ }_{K_{7} \dot{M}_{f}} \quad \dot{P}_{2}=K_{4} P_{1}-\left[K_{5} C_{d}\left(U_{e g r}\right) \Psi_{e}+K_{6} \Psi_{t}\right] P_{2}+
$$$$
\dot{X}_{0,1}=K_{2} C_{d}\left(U_{e g r}\right) \Psi_{e} \frac{P_{2}}{P_{1}}\left(X_{0,2}-X_{0,1}\right)+\frac{K_{3}}{\pi-1} \frac{P_{c}}{P_{1}}\left(X_{0, a i r}-\right.
$$

$$
\begin{aligned}
& \dot{X}_{0,2}=K_{4} \frac{P_{1}}{P_{2}}\left(X_{0,1}-X_{0,2}-K_{7} \dot{M}_{f} \frac{1}{P_{2}}\left(X_{0,2}+l_{1}\right)\right. \\
& \dot{P}_{w}=K_{8} \Psi_{t}\left[1-\pi_{t}\right] P_{2}-K_{9} P_{c}
\end{aligned}
$$

The intake oxygen mass fraction is chosen as control object, that means selecting $X_{0,1}$ as output variable, the output equation is as follows:

$$
y=X_{0,1}
$$

The state space equations of the air system consists of Eq.(2-7) are considered nonliner. By applying Taylor approximation linearization method, the non linear model can be linearized as follows:

$$
\begin{aligned}
& \Delta \dot{x}=A \Delta x+B \Delta u \\
& \Delta y=C \Delta x+D \Delta u
\end{aligned}
$$

where, $\Delta x=x-x_{0}, \Delta u=u-u_{0}$, and $\Delta y=$ $y-y_{0} ; A, B, C, D$ are the coefficient matrices of the state space model, which can be derived as follows:

$$
\begin{aligned}
& A=\left(\frac{\partial f}{\partial x^{T}}\right)_{x_{0}, u_{0}}=\left[\begin{array}{lllll}
\frac{\partial f_{1}}{\partial P_{1}} & \frac{\partial f_{1}}{\partial P_{2}} & 0 & 0 & \frac{\partial f_{1}}{\partial P_{c}} \\
\frac{\partial f_{2}}{\partial P_{1}} & \frac{\partial f_{2}}{\partial P_{2}} & 0 & 0 & 0 \\
\frac{\partial f_{3}}{\partial P_{1}} & \frac{\partial f_{3}}{\partial P_{2}} & \frac{\partial f_{3}}{\partial X_{0,1}} & \frac{\partial f_{3}}{\partial X_{0,2}} & \frac{\partial f_{3}}{\partial P_{c}} \\
\frac{\partial f_{4}}{\partial P_{1}} & \frac{\partial f_{4}}{\partial P_{2}} & \frac{\partial f_{4}}{\partial X_{0,1}} & \frac{\partial f_{4}}{\partial X_{0,2}} & 0 \\
0 & \frac{\partial f_{5}}{\partial P_{2}} & 0 & 0 & \frac{\partial f_{5}}{\partial P_{c}}
\end{array}\right]_{x_{0}, u_{0}} \\
& B=\left(\frac{\partial f}{\partial u}\right)_{x_{0}, u_{0}}=\left[\begin{array}{l}
\frac{\partial f_{1}}{\partial u_{\text {egr }}} \\
\frac{\partial f_{2}}{\partial u_{\text {egr }}} \\
\frac{\partial f_{3}}{\partial u_{\text {egr }}} \\
0 \\
0
\end{array}\right. \\
& \prod_{x_{0}, u_{0}}
\end{aligned}
$$

\section{OPTIMAL CONTROL THEORY}

The state feedback controllers for this paper are designed using the optimal control theory of linear quadratic regulator algorithm (LQR). A special case of optimal control problem which is of particular importance arises when the objective function is a quadratic function of $x$ and $u$, and the dynamic equations are linear. The resulting feedback law in this case is known as the linear

(4) quadratic regulator (LQR). The LQR controller generates the parameters of the gain by minimizing the error criteria in Eq.(10). 


\section{Available online at www.ijrat.org}

$J=\frac{1}{2} \int_{0}^{\infty}\left(x^{T} Q x+u^{T} R u\right) d t$

where $\mathrm{Q}$ and $\mathrm{R}$ are the positive-definite Hermitian or real symmetric matrices.

From the above equations,

$K=-R^{-1} B^{T} P$

and hence the control law is,

$u(t)=-K x(t)=-R^{-1} B^{T} P x(t)$

In which $\mathrm{P}$ must satisfy the reduced Riccati equation:

$P A+A^{T} P-P B R^{-1}+B^{T} P+Q=0$

The LQR function allows to choose two parameters, $\mathrm{R}$ and $\mathrm{Q}$, which will balance the relative importance of the input and state in the cost function that you are trying to optimize. Essentially, the LQR method allows for the control of all outputs.

\section{SWITCHED LINEAR CONTROL THEORY}

In control theory, the literature reveals that, the use of switching in any control applications has proved to give better performance compared to without switching in control. Switching supervisor will guide number of control structures to switch between them. The combination of continous and discrete systems are refered to as hybrid or switching systems. The multi controller architecture for switched linear systems is as shown in Fig. 2, where the switching takes place between different controllers as per the instruction of supervisor to the process.

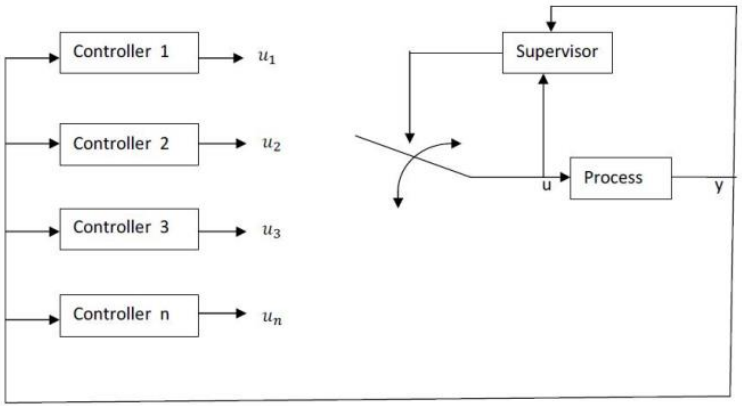

Fig. 2: Multicontroller Architecture of Switched System A switched-linear system model is as follows:

$\dot{x}(t)=A_{\sigma(t)} x(t)$

The switching signal $\sigma(t)$ indicates

$$
\begin{gathered}
\dot{x}(t)=A_{1} x(t) \text { if } \sigma(t)=1 \\
=A_{2} x(t) \text { if } \sigma(t)=2
\end{gathered}
$$

The three standard switching control algorithms which were suitably modified and implemented according to the automotive system based LTI models in the present research are briefed here.

\section{A. Switching Control Algorithm I}

The switching control algorithm I, for finding the switching matrix $S$ is as follows (Lalitha $S$ Devarakonda et al. 2006; Yathisha L \& S Patil Kulkarni et al. 2015 \& 2017):

$$
\begin{gathered}
\dot{x}(t)=\left(A-B K_{1}\right) x(t) \quad x^{\prime} S x \leq 0 \\
=\left(A-B K_{2}\right) x(t) \quad x^{\prime} S x>0
\end{gathered}
$$

1. Define $A_{1}$ has asymptotically stable system and $A_{2}$ to be may or may not be stable.

2. Determine $T_{0}$ by solving the algebraic Lyapunov Equation: $A_{1}^{T} T_{0}+T_{0} A_{1}=-C^{T} C$.

3. Find the switching matrix $S$ from the switching matrix equation: $S=-\left(A_{2}^{T} T_{0}+T_{0} A_{2}+C^{T} C\right)$.

\section{B. Switching Control Algorithm II}

The switching control algorithm II, is as follows (Kourosh Davoodi \& S Patil Kulkarni et al. 2016):

$$
\dot{x}(t)=A_{\sigma(t)} x(t)
$$

where

$$
\begin{gathered}
\sigma(t)=i, \quad \in \Omega_{i} \\
=j, \quad \notin \Omega_{j}
\end{gathered}
$$

The switching signal $\sigma(t)$ changes its value only if the state trajectory leaves one of the regions $\Omega_{i}$. It holds constant value only if the state trajectory keeps within a particular region.

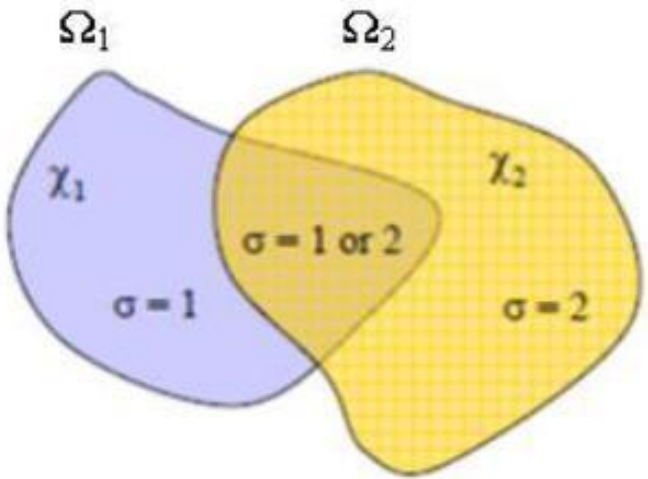

Fig. 3: State space partitioning into operating regions

The switching signal $\sigma(t)$ changes its value when the trajectory leaves one of the regions, and the next switching can occur only when the trajectory leaves the other region after having traversed the intersection $\Omega_{i} \cap \Omega_{j}$.

The controller gains for switching are designed such that, all closed loop subsystems are quadratically stable in the region where they are active. For all $x \in \Omega_{i}$ and $x \neq 0$, Lyapunov-like functions $V_{i}(x)=x^{T} P_{i} x$ are 
defined where, $P_{i} \in R^{n \times n}, P_{i}=P_{i}^{T}$ and $P_{i}>0$. The quadratic stability conditions for all subsystems are the existence of $P_{i}$ such that, $\dot{V}_{i}(x)<0$. In other words, for region,

$\Omega_{i} \equiv\left\{x \in R^{n} \mid x^{T} P_{i} x \geq 0\right\}, \forall i \in[1,2, \cdots, N](19)$

we have,

$$
x^{T}\left[A_{i}^{T} P_{i}+P_{i} A_{i}\right] x<-\epsilon \equiv A_{i}^{T} P_{i}+P_{i} A_{i}<-\epsilon
$$

For sufficiently small value of $\epsilon>0$. Quadratic stability is a special class of exponential stability, which implies asymptotic stability.

\section{A. Switching Control Algorithm III}

The switching boundary vectors $F_{1}$ and $F_{2}$ are designed using this switching control algorithm as follows (Yathisha L \& S Patil Kulkarni et al. 2016):

$$
\begin{gathered}
\dot{x}(t)=\left(A+B K_{1}\right) x(t) \quad x^{\prime} F_{1}^{\prime} F_{2} x>0 \\
=\left(A+B K_{2}\right) x(t) \quad x^{\prime} F_{1}^{\prime} F_{2} x \leq 0
\end{gathered}
$$

1. First, find an alternate controller $K_{\beta}$ such that it has $n-1$ closed loop real stable eigen values.

2. Second, from the closed loop eigenvalues of $\left(A+B K_{\beta}\right)$ select a primary controller $K_{\alpha}$ such that it has $n-2$ common eigen values of $\left(A+B K_{\alpha}\right)$ and the remaining eigen values are complex.

3. To design $F_{1}$, multiply the left side eigen value polynomials of $\left(A+B K_{\beta}\right)$ and select the coefficients of expanded polynomial in ascending powers of $s$.

4. To design $F_{2}=\left[F_{1}+\mu \omega_{2}\right], \quad \omega_{2}$ is calculated by multiplying the polynomial that is removed while designing the vector $F_{1}$ (right side eigen value) with other $(n-2)$ left eigen values by selecting $\mu<0$.

\section{EXPERIMENTAL SET UP}

The experimental set up for the current paper is carried out with following cases:

1. Case I (i): The two optimized feedback controllers $\left(K_{1} \& K_{2}\right)$ are designed for the turbocharged diesel engine model with the control input EGR. The switching control algorithm $I$ is applied for the two individual feedback controllers $\left(K_{1}\right.$ and $\left.K_{2}\right)$ to switch between them. The numerical values of $K_{1}, K_{2}$ are as follows:

$$
\begin{aligned}
& K_{1}=\left[\begin{array}{lllll}
0.156 & -0.989 & -1.943 \times 10^{-4} & -3.48 \times 10^{-5} & -4.17
\end{array}\right] \\
& K_{2}=\left[\begin{array}{lllll}
1.560 & -9.895 & -0.0019 & -3.476 \times 10^{-4} & -41.715
\end{array}\right]
\end{aligned}
$$

The switching matrix $S$ for all the five state variables are calculated from the swicthing control algorithm 1 rule.
2. Case I (ii): Switching Control algorithm II is applied for the designed optimized feedback controllers $\left(K_{1}\right.$ and $K_{2}$ ) of turbocharged diesel engine model as in Case I (i).

3. Case II: To show the effectiveness of stabilization between stable and unstable controller, switching control algorithm III is implemented to the diesel engine model. The optimized and non-optimized feedback controller gains $\left(K_{\alpha}\right.$ and $\left.K_{\beta}\right)$ and the switching boundary vectors $F_{1}$ and $F_{2}$ are given as:

$K_{\alpha}$

$=\left[\begin{array}{lllll}0.156 & -0.989 & -1.943 \times 10^{-4} & -3.48 \times 10^{-5} & -4.17\end{array}\right]$

$K_{\beta}$

$=\left[\begin{array}{lllll}0.3621 & -0.8374 & -5.2277 \times 10^{3} & -200.6376 & 27.7409\end{array}\right]$

$F_{1}$

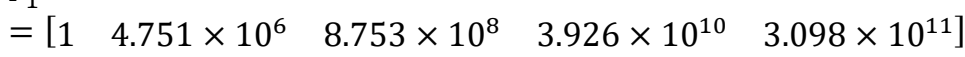

$$
F_{2}=
$$

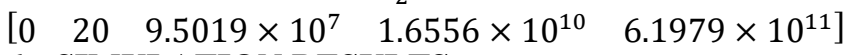

\section{SIMULATION RESULTS}

The experimental set-up to test the proposed control techniques consists of dynamic state space model described by A and B matrices below:

$A=$

$\left[\begin{array}{lllll}-130.64 & 8.91 & 0 & 0 & 1538 \\ 92.05 & -212.84 & 0 & 0 & 0 \\ -3.99 \times 10^{-5} & -8.25 \times 10^{-4} & -17.72 & 1.41 & 0.013 \\ 0.0047 & 1.80 \times 10^{-4} & 50.84 & -52.50 & 0 \\ 0 & 2.32 & 0 & 0 & -33.3\end{array}\right.$

$$
B=\left[\begin{array}{l}
1.02 * 10^{6} \\
-4.64 *^{6} \\
-94.81 \\
0 \\
0
\end{array}\right]
$$

To validate the comparison and effectiveness of proposed switching controllers the simulations are carried for all the state variables such as Intake manifold pressure deviation $\left(\Delta \dot{P}_{1}\right)$, Exhaust manifold pressure deviation $\left(\Delta \dot{P}_{2}\right)$, Oxygen mass fraction in intake manifold pressure deviation $\left(\Delta \dot{X}_{0,1}\right)$, Oxygen mass fraction in exhaust manifold pressure deviation $\left(\Delta \dot{X}_{0,2}\right)$ and Compressor power deviation $\left(\Delta \dot{P}_{w}\right)$. Fig. $4-8$, show the responses of all the five state variables of case I(i). The responses of case I (ii) are shown in Fig. 9-13. Fig. 14-18, show the responses of case II. 
Available online at www.ijrat.org

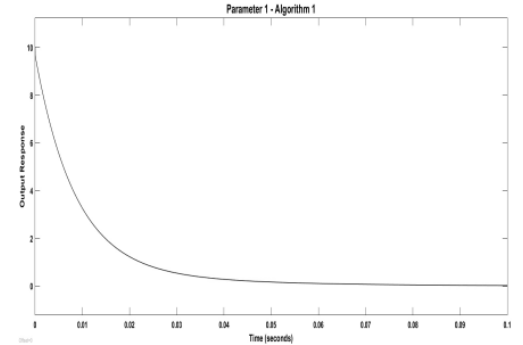

Fig. 4: Response of Case I(i) for $\Delta \dot{P}_{1}$

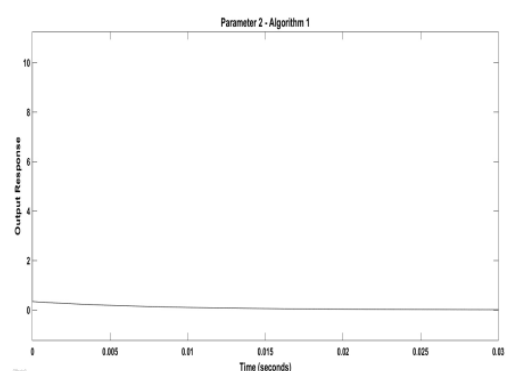

Fig. 5: Response of Case I(i) for $\Delta \dot{P}_{2}$

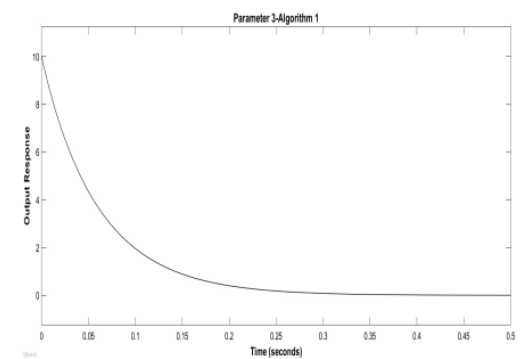

Fig. 6: Response of Case I(i) for $\Delta \dot{X}_{0,1}$

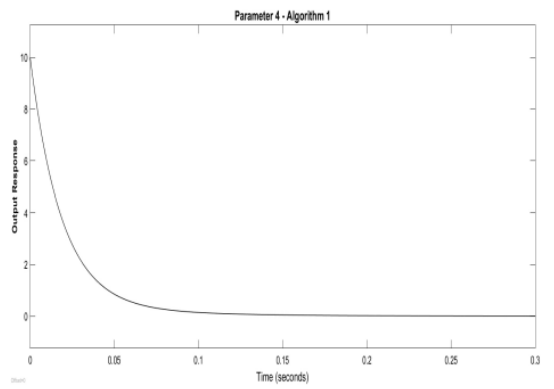

Fig. 7: Response of Case I(i) for $\Delta \dot{X}_{0,2}$

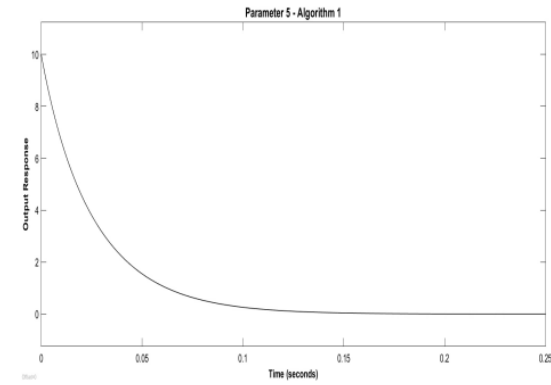

Fig. 8: Response of Case I(i) for $\Delta \dot{P}_{w}$

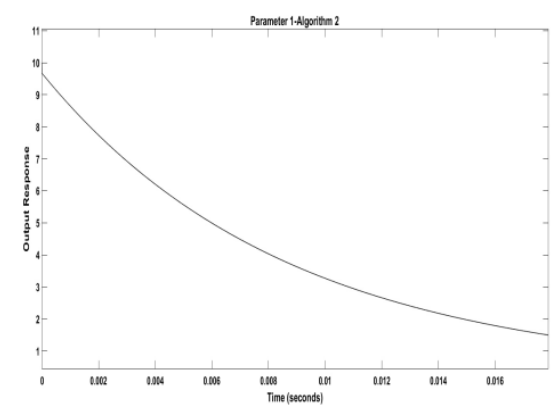

Fig. 9: Response of Case I(ii) for $\Delta \dot{P}_{1}$

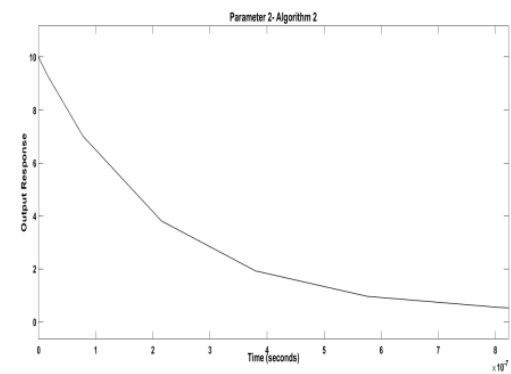

Fig. 10: Response of Case I(ii) for $\Delta \dot{P}_{2}$

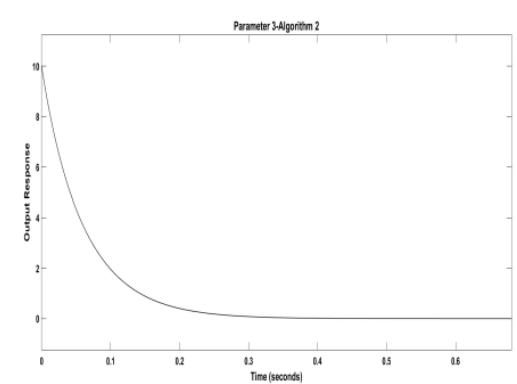

Fig. 11: Response of Case I(ii) for $\Delta \dot{X}_{0,1}$ 


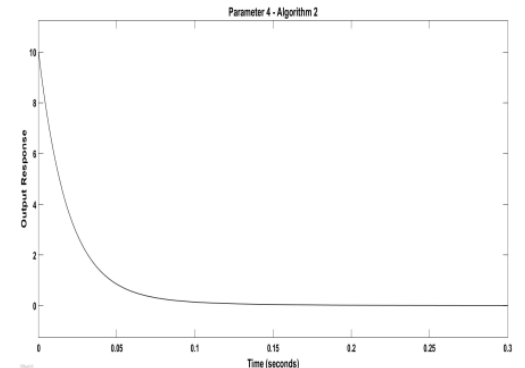

Fig. 12: Response of Case I(ii) for $\Delta \dot{X}_{0,2}$

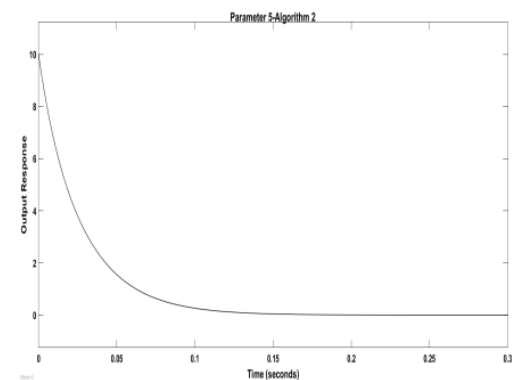

Fig. 13: Response of Case I(ii) for $\Delta \dot{P}_{w}$

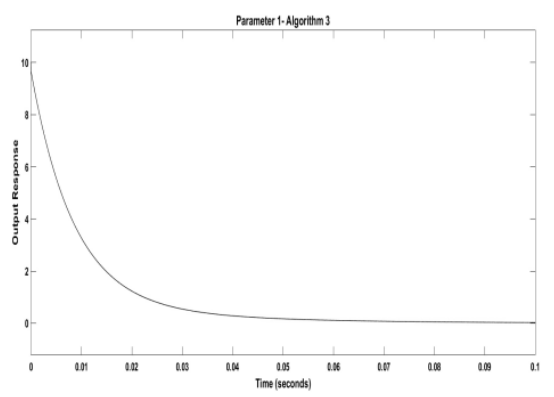

Fig. 14: Response of Case II for $\Delta \dot{P}_{1}$

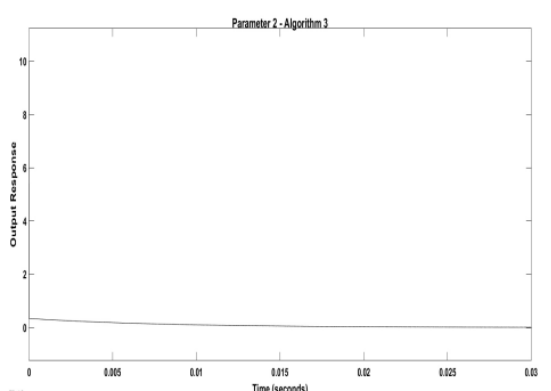

Fig. 15: Response of Case II for $\Delta \dot{P}_{2}$

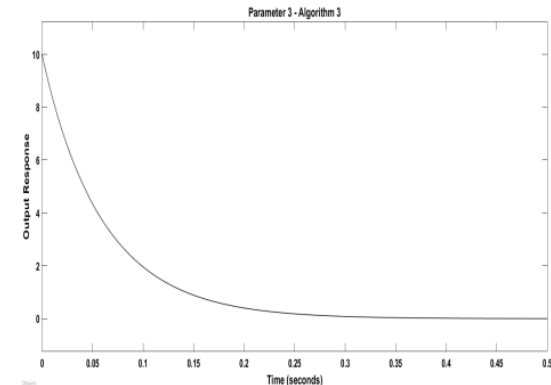

Fig. 16: Response of Case II for $\Delta \dot{X}_{0,1}$

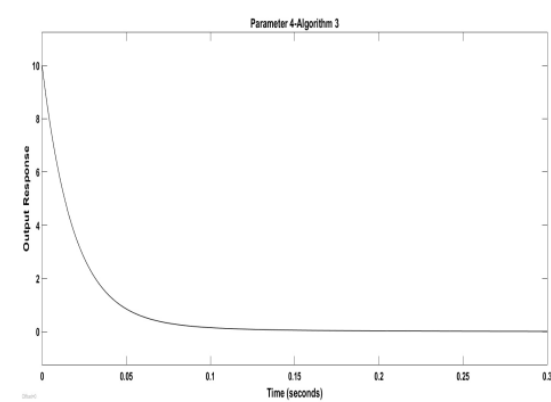

Fig. 17: Response of Case II for $\Delta \dot{X}_{0,2}$

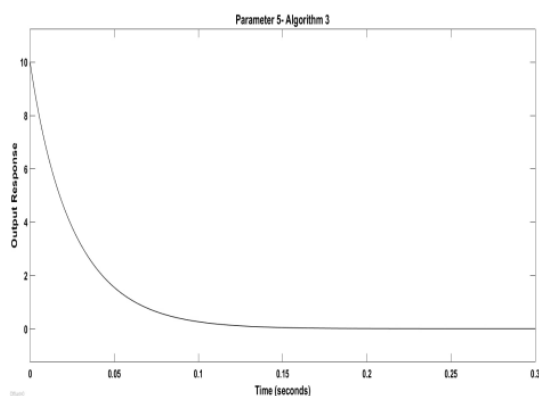

Fig. 18: Response of Case II for $\Delta \dot{P}_{w}$

Table 1: Comparison of performance index $(J=$ $\int_{0}^{\infty} y^{2} d t$ ) for Switching Algorithm I (case I(i)) and Switching Algorithm II (case I(ii))

\begin{tabular}{|c|c|c|}
\hline $\begin{array}{c}\text { State } \\
\text { Variables }\end{array}$ & $\begin{array}{c}\text { Switching } \\
\text { Algorithm I }\end{array}$ & $\begin{array}{c}\text { Switching } \\
\text { Algorithm II }\end{array}$ \\
\hline$\Delta P_{1}$ & 0.4324 & 0.4187 \\
\hline$\Delta P_{2}$ & 0.0004529 & $1.124 \times 10^{-5}$ \\
\hline$\Delta X_{0,1}$ & 2.998 & 2.998 \\
\hline$\Delta X_{0,2}$ & 0.9724 & 0.9724 \\
\hline$\Delta P_{w}$ & 1.281 & 1.281 \\
\hline
\end{tabular}

\section{DISCUSSION}

Table I, shows the comparison of optimization with respect to both the switching control algorithms. The Fig. 4-13 and Table I, indicate that the response of all the state variables are similar with respect to both the switching control algorithms except that there is slight 


\section{International Journal of Research in Advent Technology, Vol.7, No.4, April 2019 \\ E-ISSN: 2321-9637 \\ Available online at www.ijrat.org}

improvements in the state variables of $\Delta P_{1}$ and $\Delta P_{2}$ with respect to the performance index $J$ in switching algorithm II, compared to switching algorithm I.

Later, the Case II simulation results using switching control algorithm III, reveal that eventhough one of the subsystem closed loop controller eigen value is unstable, by switching between the asymptotically stable eigen value controller, the overall switched system will achieve stability and it is depicted in Fig. 14-18 for all the state variables of the turbocharged diesel engine with an EGR system.

\section{CONCLUSION}

This paper presents the application of two standard switching control algorithms with suitable modifications for the turbocharged diesel engine model with an EGR system. The two feedback controllers are derived from the optimal LQR control theory by tuning the weighting matrices $Q$ and $R$, which was used for the switching in the system. The simulations are carried for all the state variables and the results are tabulated with repect to optimization parameter $J$ (performance index) to select the best switching algorithm in future.

An intelligent existing switching stabilization algorithm with suitable modifications based on the reqirement is also applied for the two state feedback controllers (Stable and unstable). The current experiment is conducted to show that even if the system goes to unstable condition due to environment and other factors, the system can be maintained in the stable condition by switching between that unstable controller with another stable controller using the switching algorithm III. The results of evidence are shown in Fig. 14-18.

\section{REFERENCES}

[1] Magdi S. Mahmoud, "Improved Controller Design for Turbocharged Diesel Engine", Proceedings of the world Congress on Engineering, Vol. 3, July 4 - 6, 2012, London, U.K.

[2] Adrian Ilka, Nikolce Murgovski and Jonas Fredriksson, "Air-management and fueling strategy for diesel engines from multi-layercontrol perspective", $9^{\text {th }}$ IFAC International Symposium on Advances in Automotive Control, Orlans, France, 2427, 2019.

[3] Keith R. Santarelli and Munther A. Dahleh, "Comparison between a switching controller and two LTI controllers for a class of LTI plants", Int. J. Robust Nonlinear Control, 2008, pp. 1-33, DOI: 10.1002/rnc. 1308

[4] Keith R. Santarelli and Munther A. Dahleh, "L2 Gain Stability of Switched Output Feedback Controllers for a Class of LTI Systems", IEEE Transactions on Automatic Control, Vol. 54, No. 7, July 2009, pp. 1504-1514.

[5] Keith R. Santarelli and Munther A. Dahleh, "Optimal controller synthesis for a class of LTI systems via switched feedback", Systems \& Control Letters, Elsevier Journal, VOL. 59, Issue No. 3, March 2010, pp. $258-264$.
[6] Zhi Hong Huang, Cheng Xiang, Hai Lin and Tong Heng Lee, "A Necessary and Sufficient Condition for Stability of Arbitrarily Switched Second-Order LTI System: Marginally Stable Case" , 22nd IEEE International Symposium on Intelligent Control Part of IEEE Multi-conference on Systems and Control Singapore, 1-3 October 2007, pp. 83-88.

[7] Lalitha S. Devarakonda "Performance Based Switching Control for Single Input LTI Systems", M.S. Thesis, Department of Electrical Engineering, Graduate Faculty of the Louisiana State University and Agricultural and Mechanical College, Dec 2005.

[8] Jorge L. Aravena and Lalitha Devarakonda, "Performance Driven Switching Control", IEEE Transactions on Industrial Electronics, 2006, July 912, 2006, pp. 31-36.

[9] Yathisha L and S Patil Kulkarni "Application and comparison of switching control algorithms for power system stabilizer", IEEE International Conference on Industrial Instrumentation and Control (ICIC), IEEE Xplore, May 2015, Pune, pp 13001305. DOI:10.1109/IIC.2015.7150949.

[10] Yathisha L, Kourosh Davoodi and S Patil Kulkarni "Optimal switching control strategy for UPFC for wide range of operating conditions in power system", $3^{\text {rd }}$ Indian Control Conference, IEEE Xplore, Jan 2017, Indian Institute of Technology (IIT), Guwhati, $\mathrm{pp}$ 225-232. DOI:10.1109/INDIANCC.2017.7846479.

[11] Kourosh Davoodi and S Patil Kulkarni "Hybrid modeling and control of power systems with PSS operating over wide range of operating conditions", $6^{\text {th }}$ IEEE International Conference on Power Systems (ICPS-2016), IEEE Xplore, Mar 2016, New Delhi, India, pp 1-6. DOI: 10.1109/ICPES.2016.7584243.

[12] Yathisha L and S Patil Kulkarni, "Optimal Switched Feedback Controller Design for the Simultaneous Coordinated Design of UPFC and PSS in Power System", Paper accepted for publication in Journal of Electrical Engineering, POLITEHNICA, RO, Vol 2, Issue 2, 2016

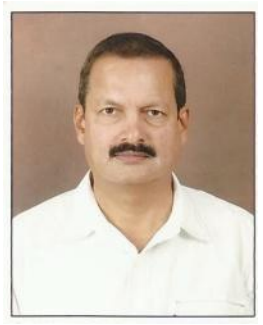

Shashidhar S Gokhale obtained his Masters Degree in Industrial Electronics from NITK, Surathkal, India, in the year 1983. Presently he is working towards his Doctoral Degree in Electronics, in the field of control systems in the prestigious University of Mysore, India. He is working as an Associate Professor in the department of Electronics and Communication Engineering, ATME College of Engineering, Mysore, India, since 2012. He has 25 years of industrial experience and 10 years of teaching experience in various industries and colleges in India as well as Singapore. 
e-mail: shashisg@gmail.com.

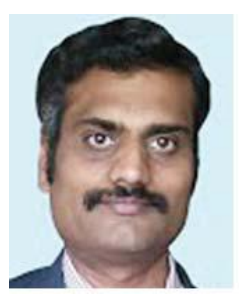

Sudarshan S Patil Kulkarni received his Ph.D. degree from Old Dominion University, Norfolk, Virginia, USA in 2004. He is currently working as Professor in the Department of Electronics \& Communication at JSSTU, Mysore, INDIA. His research interests include control systems, hybrid \& stochastic systems, VLSI, signal \& image processing. e-mail: sudarshan.pk@sjce.co.in.

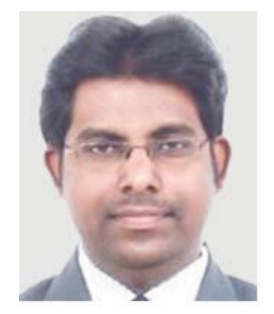

Yathisha L. received his Doctoral Degee in Control Systems \& Masters Degree in Industrial Electronics from SJCE, Visvesvaraya Technological University (VTU) in the year 2017 \& 2010 respectively. Since 2012, he is working as an Associate Professor in the Department of Electronics \& Communication Engineering, ATME College of Engineering, Mysore, INDIA. His areas of interests are Control Systems, Power Systems and Hybrid Control Systems. e-mail: yathisha.171@gmail.com. 